\title{
Influence of Different Heat Units and Supplementary Irrigation on Barley Production Under Matruh Conditions-Egypt
}

\author{
Amr.K.Mahmoud ${ }^{1} \quad$ Adel. A. Meselhy ${ }^{2}$ \\ 1.Department of Physical and soil chemistry -Desert Research Center (DRC), Cairo, EGYPT \\ 2. Department of Soil conservation and water resources -Desert Research Center (DRC), Cairo, EGYPT
}

\begin{abstract}
ABESTRACT
This study was carried out at Marsa Matruh governorate - western north coast Egypt ( $\left.31^{\circ} 15^{\prime} 35^{\prime \prime} \mathrm{N}, 27^{\circ} 9^{\prime} 43^{\prime \prime} \mathrm{E}\right)$ in the 2017/2018 growing season. The experiment was conducted to assess the different heat units or Accumulative of growing degree-days (AGDD) under various factors [sowing dates, tillage operation and different Supplementary irrigation strategies] on biomass and grain yield production for barley. Thus; the factor of sowing date comprise into three treatments $\left(15^{\text {th }}\right.$ Nov, $30^{\text {th }}$ Nov and $15^{\text {th }}$ Dec $)$. Second factor is tillage with two treatments (no tillage"NT" and tillage 20cm"T") and the third factor is Supplementary irrigation with three treatments $(0$, 70 and $140 \mathrm{~mm})$. thus; The results revealed that the $\left(1 \mathrm{Ton}^{\mathrm{F}} \mathrm{Fe}^{-1}\right)$ for $(\mathrm{BY})$ needs for $(585.3,578.73$ and 626.64$)$ heat units (AGDD) as an average under different sowing dates $\left(15^{\text {th }}\right.$ Nov, $30^{\text {th }}$ Nov and $15^{\text {th }}$ Dec.) respectively with tillage treatment " $\mathrm{T}$ ". Further; the (1 Ton.Fed $\left.{ }^{-1}\right)$ from grain yield (GY) needs for (1518) heat units if sowing on the $\left(15^{\text {th }}\right.$ Nov. $)$, for $(1531.85)$ heat units on $\left(30^{\text {th }}\right.$ Nov. $)$ and for $(1468.7)$ heat units on $\left(15^{\text {th }}\right.$ Dec.). on the hand; with no tillage (NT) the BY and GY need for more heat unit to get $\left(1 \mathrm{Ton}^{-F^{-1}}{ }^{-1}\right)$ by $(683.7,729.7$ and 807.6$)$ heat units for BY and $(1845.1,1904.1$ and 2099.5$)$ heat units for GY under different sowing date $\left(15^{\text {th }}\right.$ Nov, $30^{\text {th }}$ Nov and $15^{\text {th }}$ Dec). Notable; that the highest value for heat uses efficiency (HUE) observed with $(140 \mathrm{~mm})$ and tillage operation (T) under sowing date $15^{\text {th }}$ Nov by $(2.06 \& 0.76 \mathrm{Kg}$. fed $-1 \mathrm{C}-1$ day-1) for both (BY) and (GY) respectively. However; under sowing date 30th Nov there is not any significant differentiation for heat uses efficiency (HUE) value compare with sowing date 15 th Nov which recorded ( $2.03 \& 0.73 \mathrm{Kg}$. fed $-1 \mathrm{C}-1$ day-1) for both (BY) and (GY) respectively. On the other hand; The Aqua Crop model adequately simulated the biomass yield (BY), and grain yield (GY) for barely under different treatments. The simulated (BY) agreed well with the measured (BY) across different treatments where $\left(\mathrm{R}^{2}=0.98 \& \mathrm{E}=0.95 \& \mathrm{RMSE}=1.17 \%\right)$ for barely under different treatments of (sowing dates and supplementary irrigation strategies) with tillage process. measured and simulated (GY) were also closely related. The Aqua Crop model calibrated the GY with the prediction statistics error by $\left(\mathrm{R}^{2}=0.90 \& \mathrm{E}\right.$ $=0.51 \&$ RMSE $=1.18 \%$ ) with tillage process. Consequently; these results suggest that the good value recorded with tillage (T), (140mm) and $15^{\text {th }}$ Nov. In addition, AquaCrop model could be used to predict BY and GY of barely with a high degree of reliability at western north coast conditions. Thus; using Aqua Crop helps to determine the plantation day which records a highest efficiency for heat units and water productivity.
\end{abstract}

Keywords: Aqua Crop; Barley production; Heat unit; Sowing date; Supplementary irrigation and Tillage

DOI: $10.7176 / \mathrm{JNSR} / 9-14-04$

Publication date:July $31^{\text {st }} 2019$

\section{INTRODUCTION}

Scarcity of water and limitation water resource leads to reduction in water available for irrigation crops. In addition; Agriculture is the principal user of all water resources, such as, rainfall, water in rivers, lakes and aquifers. Thus; Rainfall is one of the most important climatic variables because of its two sided effects - as a deficient resource, such as droughts and as a catastrophic agent, such as floods. It is the primary source of water for agricultural production. For instance; Winter wheat is a vital food crop for the majority of all development country especially in Egypt. The rainfall in Egypt is $156 \mathrm{~mm}$ as an average especially at western north coast where plantation barley may be a useful for maximizing water use from rainfall.

Otherwise; Temperature is second critical parameter for climate which the potential productivity level for winter crops (Kalra et al., 2008). For most plants phonological development from seeding to maturity is related to temperature and daily accumulation of heat units. The amount of heat units required to move the plant to next development stage remains constant from year to year, however; the actual amount of time (days) can vary considerably from year to year because the change of weather conditions. For instance; barely minimum daily temperature for measurable growth is about $3.5 \mathrm{C}^{\circ}$. Mean daily temperature for optimum growth and tillering is between 15 and $20 \mathrm{C}^{\circ}$ (Doorenbos \& Kassam, 1979). Generally; barely production needs to promote all agricultural system parameters as (climate and water resource management) to get a highest value.

On the other hand; several studies have described several such irrigation strategies for use by farmers (Geerts et al., 2009). Since the mid-1960s, the relationship between water and crop yield has been described with both empirical and mechanistic models (Penning et al., 1989). Furthermore; a simulation of the soil-plant-climate continuum remains an important part of such research, especially with regard to expansion of the application range of resulting models to a wider array of cropping systems (Xiu-liang et al., 2014). 
Therefore, the Food and Agricultural Organization (FAO) developed the AquaCrop model in an effort to meet this need in 2009. This model was originated from the "yield response to water" data (Doorenbos \& Kassam, 1979)., and evolved to a normalized crop water productivity (NCWP) concept (Steduto et al.,2009). Compared with other models, AquaCrop is relatively simple to operate by those with little or no research experience, and allows for simulation of crop performance in multiple scenarios. Moreover; to a high level of accuracy, this robust model requires a limited set of input parameters, most of which are relatively easy to acquire (Hsiao et al., 2009). The AquaCrop model is also capable of predicting crop productivity, water requirements, and water use efficiency under water-limiting conditions (Raes et al ., 2009). So; the aims of this study to different heat units or Accumulative of growing degree-days (AGDD) under various sowing dates, tillage process and different water applied strategies on biomass and grain yield production for barely. Moreover; the relation between different growing degree-days (GDD) and yield production. Finally; using Aqua Crop as assimilation model to get relation between prediction and actual production.

\section{MATERIAL AND METHOD}

Study site

field experiment were conducted in the 2017/2018growing season at western north coast experimental site $\left(31^{\circ}\right.$ $\left.15^{\prime} 35^{\prime \prime} \mathrm{N}, 27^{\circ} 9^{\prime} 43^{\prime \prime} \mathrm{E}\right)$, Marsa Matruh governorate, Egypt. The site of experiment falls into an arid area with a Mediterranean climate. The site is about $92 \mathrm{~m}$ above sea level with an annual rainfall of $133.55 \mathrm{~mm} / \mathrm{year}$, temperatures of $20.7^{\circ} \mathrm{C}$, relative humidity of $65 \%$, and wind speed of $3.5 \mathrm{~m} / \mathrm{s}$. The total annual evapo-transpiration (ETo) is $1590 \mathrm{~mm} /$ year (table 1).

Table 1: Climatic characteristics at the experiment site.

\begin{tabular}{cccccccc}
\hline \multirow{2}{*}{ Month } & Prc. & Tem. $\mathbf{~ m a x}$ & Tem $\mathbf{~ m i n . ~}$ & Hum. & Sun shine & Wind (2m) & ETo \\
\cline { 2 - 8 } & $\mathbf{m m} / \mathbf{m}$ & ${ }^{\circ} \mathbf{C}$ & ${ }^{\circ} \mathbf{C}$ & $\mathbf{\%}$ & $\mathbf{\%}$ & $\mathbf{m} / \mathbf{s}$ & $\mathbf{m m} / \mathbf{d}$ \\
\hline Jan & 35.3 & 18.5 & 10.1 & 68 & 63 & 3.4 & 2.4 \\
\hline Feb & 23.2 & 21.1 & 11.7 & 67 & 63 & 4 & 3.2 \\
\hline Mar & 9.6 & 23.4 & 12.4 & 66 & 63 & 4 & 4 \\
\hline Apr & 3.6 & 24.3 & 14.2 & 63 & 68 & 4 & 4.85 \\
\hline may & 1.55 & 25.5 & 16.7 & 62 & 68 & 3.9 & 5.4 \\
\hline Jun & 0 & 28.7 & 20 & 64 & 69 & 3.5 & 5.86 \\
\hline Jul & 0 & 30.4 & 22.1 & 64 & 72 & 3.5 & 6.2 \\
\hline Aug & 0 & 30.7 & 22.1 & 64 & 72 & 3.4 & 5.94 \\
\hline Sep & 0.3 & 29.2 & 21.9 & 65 & 67 & 3.4 & 5.09 \\
\hline Oct & 10 & 25.9 & 19.5 & 67 & 55 & 3.2 & 3.8 \\
\hline Nov & 19 & 23.1 & 13.1 & 68 & 53 & 3.1 & 2.88 \\
\hline Dec & 31 & 20.4 & 12.3 & 65 & 51 & 3.2 & 2.58 \\
\hline
\end{tabular}

(Prc. = Precipitation; Tmp. $\min / \max =\operatorname{minimum} /$ maximum temperature; hum.$=$ relative humidity; Sun shine $=$ Sun shine as percentage of day length; Wind $(2 \mathrm{~m})=$ wind speed at $2 \mathrm{~m} ;$ ETo $=$ Reference evapotranspiration) (FAO AQUASTAT 2018).

Soil data analysis:

The soil at the experimental site represents the major soil type (loamy sand). Analyses of soil and some physical and chemical characteristics were carried out according to (Klute, 1986). These analyses are presented in tables [(2) and (3)]. The maximum field capacity for soil was (16.2\% at $0.0-0.2 \mathrm{~m}$ and $19 \%$ at $0.2-0.4 \mathrm{~m})$. The physical soil characteristics were measured and used for input into AquaCrop.

Table 2. Some physical characteristic and mechanical analysis for experimental site.

\begin{tabular}{cccccccccc}
\hline $\begin{array}{c}\text { Soil } \\
\text { depth } \\
(\mathbf{c m})\end{array}$ & $\begin{array}{c}\text { Bulk } \\
\text { density } \\
(\mathbf{M g} / \mathbf{c m})\end{array}$ & $\begin{array}{c}\text { Very } \\
\text { coarse } \\
\text { sand \% }\end{array}$ & $\begin{array}{c}\text { Coarse } \\
\text { sand } \\
\%\end{array}$ & $\begin{array}{c}\text { Medium } \\
\text { sand } \\
\%\end{array}$ & $\begin{array}{c}\text { Fine } \\
\text { sand } \\
\%\end{array}$ & $\begin{array}{c}\text { Very } \\
\text { fine } \\
\text { sand } \\
\%\end{array}$ & $\begin{array}{c}\text { Silt } \\
\text { +clay } \\
\%\end{array}$ & $\begin{array}{c}\text { Soil } \\
\text { Field } \\
\text { capacity } \\
\%\end{array}$ & Texture \\
\hline $\mathbf{0 - 2 0}$ & 1.62 & 0.82 & 2.69 & 34.94 & 29.49 & 29.39 & 2.67 & 16.2 & L S* \\
\hline $\mathbf{2 0 - 4 0}$ & 1.64 & 0.90 & 2.82 & 31.38 & 26.73 & 34.64 & 3.53 & 19 & L S* \\
\hline
\end{tabular}

*L.S= Loamy sand

Table 3. Some chemical characteristic for the experimental site

\begin{tabular}{cccccc}
\hline $\begin{array}{c}\text { Soil depth } \\
(\mathbf{c m})\end{array}$ & $\mathbf{O . M} \%$ & $\mathbf{N}$ & $\mathbf{P}$ & $\mathbf{K}$ & $\mathrm{Ca} \mathrm{CO}_{\mathbf{3}}$ \\
\hline $0-20$ & 0.18 & 6.8 & 12 & 0.21 & 4.63 \\
\hline $20-40$ & 0.48 & 4.2 & 10 & 0.36 & 6.74 \\
\hline
\end{tabular}

For monitoring the influence of heat unit the experiment built depending on, spilt spilt plot design, three 
factors: first factor is sowing date which divided into three treatments (Nov. $15^{\text {st }}$, Nov. $30^{\text {th }}$ and Dec. $15^{\text {th }}$ ) for barely. Second factor is tillage with two treatments (no tillage and tillage $20 \mathrm{~cm}$ ) and the third factor is Supplementary irrigation with three treatments $(0,70$ and $140 \mathrm{~mm})$ this done by adding $(70 \mathrm{~mm})$ on mid of February plus adding $(70 \mathrm{~mm})$ on the first week of March]. Further; Using machine with $180 \mathrm{~cm}$ working width Consist of seven shanks with chisel blade arranged in two rows and forward speed of tractor was $4.5 \mathrm{~km} \mathrm{~h}^{-1}$ for implement the tillage processes. Memorable; that the harvest was accomplish on mid of April.

\section{Growing degree-days (heat units) (GDD)}

Growing degree days (GDD) or heat units was calculated using the single sine curve method (Baskerville \& Emin, 1969) during growing season of barely crop. This simple linear method requires only daily minimum and maximum air temperatures, which recorded by the local meteorological weather station in site of experiment, equation (1) give explanation for calculating growing degree days:

Where:

$$
\mathrm{GDD}=\left[\left(\mathrm{T}_{\max }+\mathrm{T}_{\min }\right) / 2\right]-\mathrm{T}_{\text {base }}
$$

$\mathrm{T}_{\max } \quad=\quad$ Daily maximum temperature $\left(\mathrm{C}^{\circ}\right)$

$\mathrm{T}_{\min } \quad=\quad$ Daily minimum temperature $\left(\mathrm{C}^{\circ}\right)$, and

$\mathrm{T}_{\text {base }} \quad=\quad$ Base temperature $\left(\mathrm{C}^{\circ}\right)$

Heat use efficiency (HUE) is the ration of yield to accumulated growing degree days according to (Kingra \& Prabhjyot-Kaur, 2012) equation ( 2).

Where:

$$
\text { HUE }=\text { Yield(Ygi) / (AGDD) }
$$

$$
\begin{array}{lll}
\text { HUE } & = & \text { Heat use efficiency }\left(\mathrm{kg} \mathrm{fed}^{-1} \mathrm{C}^{\circ-1} \text { day }^{-1}\right) \\
\mathrm{Y}_{\mathrm{gi}} & = & \text { The economic yield }(\mathrm{kg} / \mathrm{fed}) . \\
\text { GDD } & = & \text { Accumulated growing degree days }\left(\mathrm{C}^{\circ} \text { day }\right) .
\end{array}
$$

Heat units are often used to predict the rate of phonological development of plant species. Developmental rates increase approximately linearly as a function of air temperature (Snyder et al., 1999), therefore the higher or lower temperature will be affected on crop by reducing the plant growth and total yield. So; the lower temperature ( $\mathrm{T}_{\text {base }}$ ), was set as $3.5 \mathrm{C}^{\circ}$ (Morteza, et al 2015; Bishnoi, et al , 1995).

\section{Clarification AquaCrop Model}

The AquaCrop model was proposed by the FAO in 2009, with a detailed description presented in (Steduto et al., 2009) and ( Raes et al.,2009a). The model computes a daily water balance, and separates evapotranspiration into evaporation and transpiration components. The crop's stomata conductance, canopy senescence, leaf growth, and yield response to water stress are modelled using four stress coefficients (stomata closure, leaf expansion, canopy senescence, and change in harvest index (Hi). The model subsequently estimates yield from the daily crop transpiration values. (fig.1)-(Dirk.et al.,2011)

Moreover; Some of the advantages of AquaCrop are: a) it is widely applicable with acceptable accuracy; b) it requires only commonly available input (i.e. climate, soil, crop and field data); all these input data were used in the model to predict the yield, water productivity, biomass and harvest index of a given crop c) it allows easy verification of simulation results with simple field observations. In general, the crop water productivity (CWP) is considered constant for a given climate condition and crop (For crops not nutrient-limited, the model provides categories ranging from slight to severe deficiencies corresponding to lower water productivity (WP)). So; the CWP remained at $1.5 \mathrm{~g} \mathrm{~m}^{-2}$ for the barely (Johannes et al., 2015). Moreover; the crop's daily aboveground biomass is calculated using CWP from the AquaCrop model (Hsiao et al., 2009) 


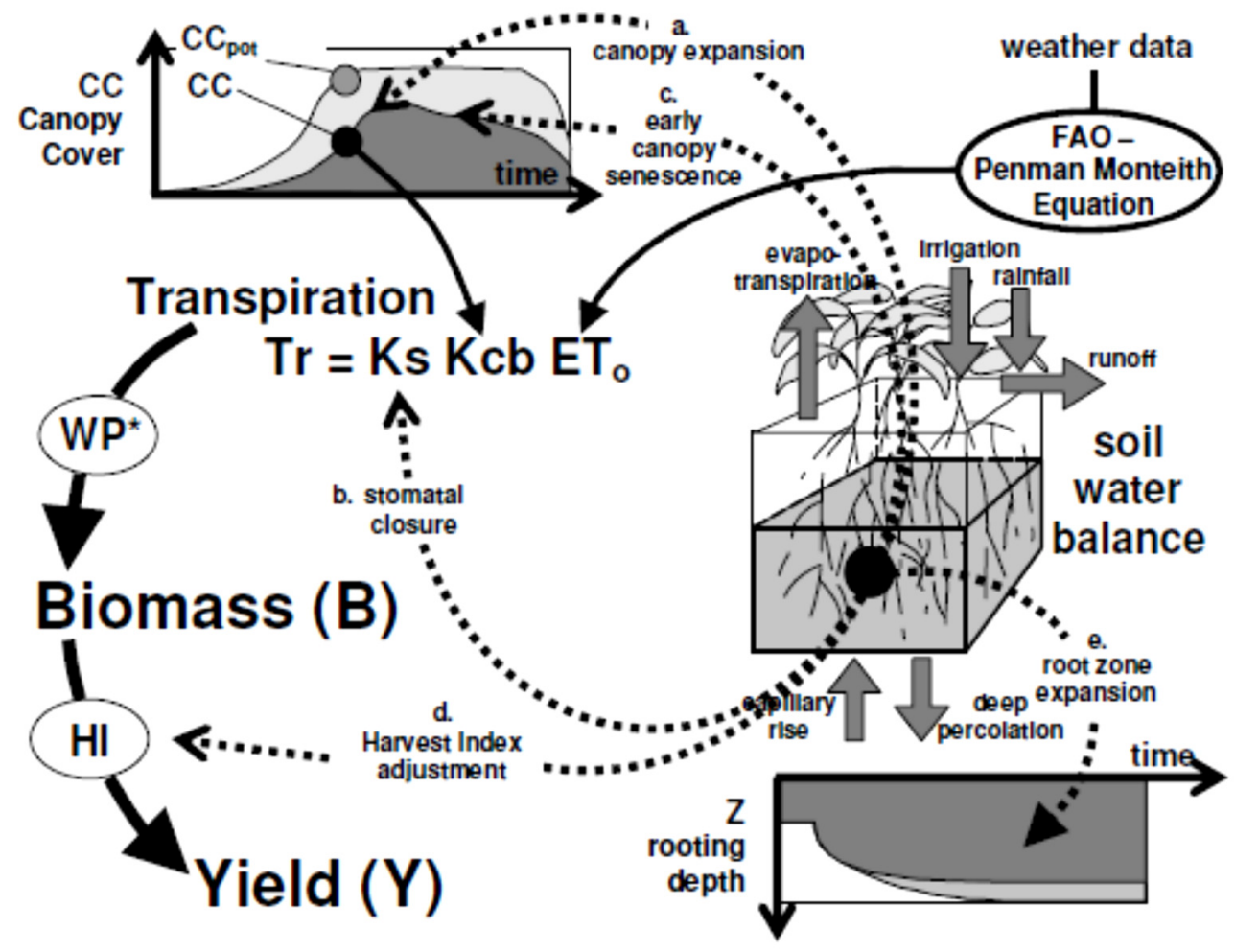

Figure 1. Flowchart calculation scheme for AquaCrop with indication of the processes and main components of soil-plant-atmosphere

Biomass yield (BY) is calculated by multiplying CWP by the ratio of crop transpiration (T), and evapotranspiration (ETo), following calculation of BY (its harvestable portion), and the grain yield (GY) is determined via harvest index (Hi).

Where:

$$
\begin{gathered}
\mathrm{BY}=\sum\left(\frac{T}{E T_{o}}\right) \times C W P \\
\mathrm{GY}=\mathrm{BY} x \mathrm{Hi}
\end{gathered}
$$

$\begin{array}{lll}\mathrm{BY} & = & \text { Biomass Yield }\left(\mathrm{Kg} . \mathrm{Fed}^{-1}\right) \text {, and } \\ \mathrm{T} & = & \text { Crop transpiration }(\mathrm{mm}) \text {, and } \\ \mathrm{ET}_{\mathrm{o}} & = & \text { Reference evapotranspiration }(\mathrm{mm}) \text {, and } \\ \mathrm{CWP} & = & \text { Crop water productivity }\left(\mathrm{g} \mathrm{m}^{-2}\right) . \\ \mathrm{GY} & = & \text { Grain yield }\left(\mathrm{Kg}_{\text {. Fed }}{ }^{-1}\right) \\ \mathrm{Hi} & = & \text { harvest index. }\end{array}$

The coefficient of determination $\left(\mathrm{R}^{2}\right)$, root mean square error (RMSE), and model efficiency (E) were used as the error statistics to evaluate both calibration and validation results. These statistical indices were used to compare measured and simulated values. Model performance was assessed using E (Nash and Sutcliffe, 1970) as follows:

$$
\begin{gathered}
\mathrm{E}=1-\frac{\sum_{\mathrm{i}=1}^{\mathrm{n}}\left(\mathrm{S}_{\mathrm{i}}-\mathrm{O}_{\mathrm{i}}\right)^{2}}{\sum_{\mathrm{i}=1}^{\mathrm{n}}\left(\mathrm{O}_{\mathrm{i}}-\overline{\mathrm{O}}_{\mathrm{i}}\right)^{2}} \\
\mathrm{RMSE}=\sqrt{\frac{\sum_{\mathrm{i}=1}^{\mathrm{n}}\left(\mathrm{S}_{\mathrm{i}}-\mathrm{O}_{\mathrm{i}}\right)^{2}}{n}}
\end{gathered}
$$

Where:

$$
\begin{aligned}
& \mathrm{Si}=\text { Predicted data, and } \\
& \mathrm{Oi}=\text { Observed data, and } \\
& \overline{\mathrm{O}} \mathrm{i}=\text { Mean value of } \mathrm{Oi} \text {, and } \\
& \mathrm{N} \quad=\quad \text { Number of observation. }
\end{aligned}
$$

When $\mathrm{E}$ and $\mathrm{R}^{2}$ approaching one, and a RMSE near zero this indicate that the model performance were improved.

Finally; The data were analyzed using the three way ANOVA as spilt split plot with Duncan's HSD test at $\mathrm{p}<0.05$ using the COSTAT 3.03 System software. 


\section{RESULT AND DISCUSSION}

Accumulated growing degree days (AGDD):

Table (4) illustrate the mean 10 day monthly, real and adjusted temperature, growing degree days (GDD) and accumulated growing degree days (AGDD) during barely growing season; clearly, the total amount of heat units required for barely to develop from one point to another in its life cycle was $1812.5,1668.7$ and $1646 \mathrm{C} \%$ season, for sowing date $15^{\text {th }}$ Nov, $30^{\text {th }}$ Nov and $15^{\text {th }}$ Dec. respectively. As shown at fig (2); with no tillage treatment the biomass yield for barely has significant different value for both $(70 \mathrm{~mm}$ and 140mm) under different AGDD 1812.5, 1668.7 and $1646 \mathrm{c}^{\circ}$ day. However; the biomass values under $(0 \mathrm{~mm})$ recorded a lowest value comparing with other water treatment by $\left(2.4,2.3\right.$ and 1.99 ton.fed-1) under $\left(1812.5,1668.7\right.$ and $1646 \mathrm{c}^{\circ} /$ season) respectively. Meaning that the (1 Ton.fed-1) Biomass Yield from barley needs for $(585.3,578.7$ and 626.6) heat units as an average under different sowing dates $\left(15^{\text {th }}\right.$ Nov, $30^{\text {th }}$ Nov and $15^{\text {th }}$ Dec.) respectively with different treatments. Clearly; under tillage the biomass increases liner with increasing the AGDD. This relation can be summarizing by (Eq.7).

Where: -

$$
\mathrm{BY}_{\mathrm{nt}}=0.0023(\mathrm{AGDD})-1.1406
$$

$\mathrm{BY}_{\mathrm{nt}} \quad=$ The Biomass yield for wheat under No tillage $\left(\right.$ Ton. fed $\left.{ }^{-1}\right)$.

$\mathrm{AGDD}=$ Accumulative of growing degree-days $\left(\mathrm{c}^{\circ}\right.$ day)

On the other hand; under No tillage the biomass values have different demeanour; where, there is an influence for AGDD on Biomass with high amount of Supplementary irrigation (140mm). In addition; the value recorded (2.651 ton.fed-1) with (1812.5 $\mathrm{c}^{\circ} /$ season) and (2.038 ton.fed-1) with (1646 $\mathrm{c}^{\circ} /$ season). Further; with $(70 \mathrm{~mm})$ biomass's value recorded a significant impact comparing with $(0 \mathrm{~mm})$ by $(0.76$ Ton.Fed-1) as an average under different sawing dates. Notable; that the (1 Ton.Fed-1) biomass yield needs for $(683.7,729.7$ and 807.6) heat units as an average under different sowing dates $\left(15^{\text {th }}\right.$ Nov, $30^{\text {th }}$ Nov and $15^{\text {th }}$ Dec $)$ respectively. The flowing equation (Eq.8) represented the relation between (AGDD) and (BY) with No tillage under such conditions.

Where: -

$$
\mathrm{BY}_{\mathrm{t}}=0.0033(\mathrm{AGDD})-3.2685
$$

$\mathrm{BY}_{\mathrm{t}} \quad=$ The Biomass yield for wheat under tillage $\left(\right.$ Ton. fed $\left.{ }^{-1}\right)$.

$\mathrm{AGDD}=$ Accumulative of growing degree-days $\left(\mathrm{c}^{\circ}\right.$ day $)$


Tillage

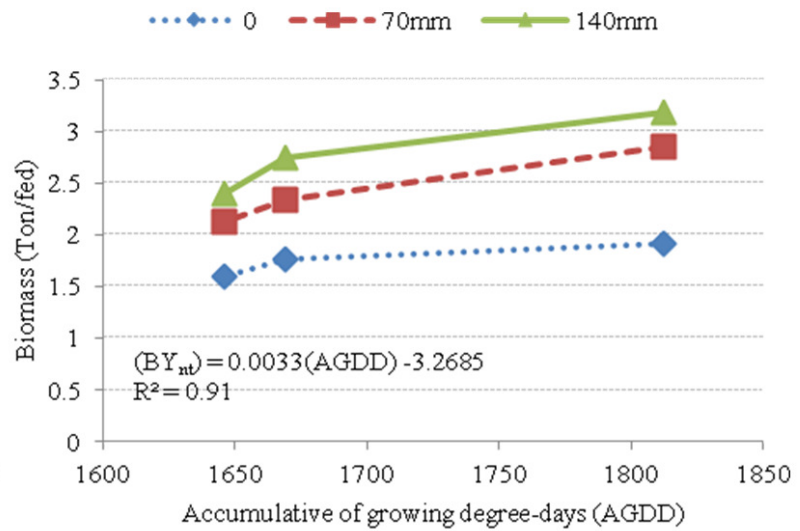

No Tillage

Figure 2. Relationship between Biomass Yield (BY) for barley and Accumulative of growing degree-days under different treatments. 


\begin{tabular}{|c|c|c|c|c|c|c|c|c|c|c|c|c|c|c|c|}
\hline \multirow[b]{2}{*}{ Months } & \multicolumn{5}{|c|}{ sowing date $15^{\text {th }}$ Nov. } & \multicolumn{5}{|c|}{ sowing date $30^{\text {th }}$ Nov. } & \multicolumn{5}{|c|}{ sowing date $15^{\text {th }}$ Dec. } \\
\hline & $\begin{array}{c}\text { Dat } \\
\mathrm{e}\end{array}$ & $\begin{array}{l}\mathrm{T}_{\mathrm{m}} \\
\mathrm{ax}\end{array}$ & $\begin{array}{c}T_{m i} \\
n\end{array}$ & $\begin{array}{c}\text { GD } \\
\text { D }\end{array}$ & $\begin{array}{l}\mathrm{AG} \\
\mathrm{DD}\end{array}$ & $\begin{array}{c}\text { Dat } \\
\text { e }\end{array}$ & $\begin{array}{c}\mathrm{T}_{\mathrm{ma}} \\
\mathrm{x}\end{array}$ & $\begin{array}{c}T_{m i} \\
n\end{array}$ & $\begin{array}{c}\text { GD } \\
\text { D }\end{array}$ & $\begin{array}{l}\mathrm{AG} \\
\mathrm{DD}\end{array}$ & $\begin{array}{c}\text { Dat } \\
\text { e }\end{array}$ & $\begin{array}{l}\mathrm{T}_{\mathrm{m}} \\
\mathrm{ax}\end{array}$ & $\begin{array}{c}T_{m i} \\
n\end{array}$ & $\begin{array}{c}\text { GD } \\
\text { D }\end{array}$ & $\begin{array}{l}\mathrm{AG} \\
\mathrm{DD}\end{array}$ \\
\hline $\begin{array}{c}2017- \\
2018 \\
\end{array}$ & $\mathrm{D}$ & $\mathrm{C}^{\circ}$ & $\mathrm{C}^{\circ}$ & $\mathrm{C}^{\circ}$ & $\begin{array}{c}\mathrm{C}^{\circ} \\
\text { day }\end{array}$ & D & $\mathrm{C}^{\circ}$ & $\mathrm{C}^{\circ}$ & $\mathrm{C}^{\circ}$ & $\begin{array}{c}\mathrm{C}^{\circ} \\
\text { day }\end{array}$ & $\mathrm{D}$ & $\mathrm{C}^{\circ}$ & $\mathrm{C}^{\circ}$ & $\mathrm{C}^{\circ}$ & $\begin{array}{c}\mathrm{C}^{\circ} \\
\text { day }\end{array}$ \\
\hline \multirow[t]{2}{*}{ Nov } & $\begin{array}{l}15- \\
24\end{array}$ & $\begin{array}{c}22 . \\
9\end{array}$ & $\begin{array}{c}12 . \\
7\end{array}$ & 143 & 143 & $*$ & $*$ & $*$ & * & * & $*$ & $*$ & * & * & $*$ \\
\hline & $\begin{array}{c}25- \\
4 \\
\end{array}$ & $\begin{array}{c}21 . \\
8\end{array}$ & $\begin{array}{c}11 . \\
6 \\
\end{array}$ & 132 & 275 & * & $*$ & $*$ & * & * & * & $*$ & $*$ & $*$ & $*$ \\
\hline \multirow{3}{*}{ Dec } & $\begin{array}{l}5- \\
14\end{array}$ & 20 & $\begin{array}{c}12 . \\
6\end{array}$ & 128 & 368 & $\begin{array}{c}30- \\
9\end{array}$ & $\begin{array}{c}20 . \\
7\end{array}$ & $\begin{array}{c}11 . \\
7\end{array}$ & 127 & 127 & * & $*$ & $*$ & * & $*$ \\
\hline & $\begin{array}{l}15- \\
24\end{array}$ & $\begin{array}{c}20 . \\
8\end{array}$ & $\begin{array}{c}14 . \\
1\end{array}$ & $\begin{array}{c}139 \\
.5\end{array}$ & $\begin{array}{c}507 . \\
5\end{array}$ & $\begin{array}{l}10- \\
19\end{array}$ & $\begin{array}{c}21 . \\
2\end{array}$ & $\begin{array}{c}12 . \\
8\end{array}$ & 135 & 262 & $\begin{array}{l}15- \\
24\end{array}$ & $\begin{array}{c}20 . \\
8\end{array}$ & $\begin{array}{c}14 . \\
1\end{array}$ & $\begin{array}{c}139 \\
.5\end{array}$ & $\begin{array}{c}139 . \\
5\end{array}$ \\
\hline & $\begin{array}{c}25- \\
3 \\
\end{array}$ & $\begin{array}{c}18 . \\
3\end{array}$ & $\begin{array}{c}10 . \\
6 \\
\end{array}$ & $\begin{array}{c}109 \\
.5 \\
\end{array}$ & 617 & $\begin{array}{c}20- \\
29 \\
\end{array}$ & $\begin{array}{c}19 . \\
9 \\
\end{array}$ & $\begin{array}{c}12 . \\
9\end{array}$ & 129 & 391 & $\begin{array}{c}25- \\
3 \\
\end{array}$ & $\begin{array}{c}18 . \\
3 \\
\end{array}$ & $\begin{array}{c}10 . \\
6\end{array}$ & $\begin{array}{c}109 \\
.5\end{array}$ & 249 \\
\hline \multirow{3}{*}{ Jan } & $\begin{array}{l}4- \\
13\end{array}$ & $\begin{array}{c}19 . \\
4\end{array}$ & 9.9 & $\begin{array}{c}111 \\
.5\end{array}$ & $\begin{array}{c}693 . \\
5\end{array}$ & $\begin{array}{c}30- \\
8\end{array}$ & $\begin{array}{c}18 . \\
8\end{array}$ & $\begin{array}{c}10 . \\
5\end{array}$ & $\begin{array}{c}111 \\
.5\end{array}$ & $\begin{array}{c}502 . \\
5\end{array}$ & $\begin{array}{l}4- \\
13\end{array}$ & $\begin{array}{c}19 . \\
4\end{array}$ & 9.9 & $\begin{array}{c}111 \\
.5\end{array}$ & $\begin{array}{c}360 . \\
5\end{array}$ \\
\hline & $\begin{array}{l}14- \\
23\end{array}$ & $\begin{array}{c}19 . \\
4\end{array}$ & 9.9 & $\begin{array}{c}111 \\
.5\end{array}$ & 805 & $\begin{array}{l}9- \\
18\end{array}$ & $\begin{array}{c}19 . \\
5\end{array}$ & 9.9 & 112 & $\begin{array}{c}579 . \\
5\end{array}$ & $\begin{array}{l}14- \\
23\end{array}$ & $\begin{array}{c}19 . \\
4\end{array}$ & 9.9 & $\begin{array}{c}111 \\
.5\end{array}$ & 472 \\
\hline & $\begin{array}{c}24- \\
2 \\
\end{array}$ & 16 & 10 & 95 & 900 & $\begin{array}{l}19- \\
28\end{array}$ & $\begin{array}{c}17 . \\
4\end{array}$ & 9.8 & 101 & $\begin{array}{c}680 . \\
5 \\
\end{array}$ & $\begin{array}{c}24- \\
2\end{array}$ & 16 & 10 & 95 & 567 \\
\hline \multirow{4}{*}{ Feb } & $3-$ & 21. & 13. & 138 & 1003 & $29-$ & 21. & 12. & 134 & & $3-$ & 21. & 13. & 138 & 705. \\
\hline & 12 & 5 & 2 & .5 & .5 & 7 & 7 & 2 & .5 & 5 & 12 & 5 & 2 & .5 & 5 \\
\hline & $\begin{array}{l}13- \\
22\end{array}$ & $\begin{array}{c}20 . \\
2\end{array}$ & $\begin{array}{c}10 . \\
5\end{array}$ & $\begin{array}{c}118 \\
.5\end{array}$ & 1122 & $\begin{array}{l}8- \\
17\end{array}$ & $\begin{array}{c}19 . \\
6\end{array}$ & $\begin{array}{c}11 . \\
8\end{array}$ & 122 & 902 & $\begin{array}{l}13- \\
22\end{array}$ & $\begin{array}{c}20 . \\
2\end{array}$ & $\begin{array}{c}10 . \\
5\end{array}$ & $\begin{array}{c}118 \\
.5\end{array}$ & 824 \\
\hline & $\begin{array}{c}23- \\
4 \\
\end{array}$ & 23 & $\begin{array}{c}11 . \\
8 \\
\end{array}$ & 139 & 1261 & $\begin{array}{l}18- \\
27 \\
\end{array}$ & $\begin{array}{c}21 . \\
8\end{array}$ & $\begin{array}{c}11 . \\
5\end{array}$ & $\begin{array}{c}131 \\
.5 \\
\end{array}$ & $\begin{array}{c}1033 \\
.5 \\
\end{array}$ & $\begin{array}{c}23- \\
4 \\
\end{array}$ & 23 & $\begin{array}{c}11 . \\
8\end{array}$ & 139 & 963 \\
\hline \multirow{3}{*}{ Mar. } & $\begin{array}{l}5- \\
14\end{array}$ & $\begin{array}{c}23 . \\
5\end{array}$ & $\begin{array}{c}13 . \\
3\end{array}$ & 149 & 1375 & $\begin{array}{c}28- \\
9\end{array}$ & 25 & $\begin{array}{c}13 . \\
4\end{array}$ & 157 & $\begin{array}{c}1190 \\
.5\end{array}$ & $\begin{array}{l}5- \\
14\end{array}$ & $\begin{array}{c}23 . \\
5\end{array}$ & $\begin{array}{c}13 . \\
3\end{array}$ & 149 & 1112 \\
\hline & $\begin{array}{l}15- \\
24\end{array}$ & $\begin{array}{c}24 . \\
4\end{array}$ & $\begin{array}{c}12 . \\
3\end{array}$ & $\begin{array}{c}148 \\
.5\end{array}$ & $\begin{array}{c}1523 \\
.5\end{array}$ & $\begin{array}{c}10- \\
19\end{array}$ & $\begin{array}{c}22 . \\
2\end{array}$ & $\begin{array}{c}11 . \\
4\end{array}$ & 133 & $\begin{array}{c}1288 \\
.5\end{array}$ & $\begin{array}{l}15- \\
24\end{array}$ & $\begin{array}{c}24 . \\
4\end{array}$ & $\begin{array}{c}12 . \\
3\end{array}$ & $\begin{array}{c}148 \\
.5\end{array}$ & $\begin{array}{c}1260 \\
.5\end{array}$ \\
\hline & $\begin{array}{c}25- \\
3 \\
\end{array}$ & $\begin{array}{c}21 . \\
7 \\
\end{array}$ & $\begin{array}{c}11 . \\
4\end{array}$ & $\begin{array}{c}130 \\
.5 \\
\end{array}$ & 1654 & $\begin{array}{c}20- \\
29 \\
\end{array}$ & $\begin{array}{c}24 . \\
1 \\
\end{array}$ & $\begin{array}{c}13 . \\
1 \\
\end{array}$ & 151 & $\begin{array}{c}1439 \\
.5 \\
\end{array}$ & $\begin{array}{c}25- \\
3 \\
\end{array}$ & $\begin{array}{c}21 . \\
7 \\
\end{array}$ & $\begin{array}{c}11 . \\
4 \\
\end{array}$ & $\begin{array}{c}130 \\
.5 \\
\end{array}$ & 1391 \\
\hline \multirow{2}{*}{ April } & $\begin{array}{l}4- \\
13\end{array}$ & $\begin{array}{c}23 . \\
7\end{array}$ & $\begin{array}{c}13 . \\
8\end{array}$ & $\begin{array}{c}152 \\
.5\end{array}$ & $\begin{array}{c}1771 \\
.5\end{array}$ & $\begin{array}{c}30- \\
8\end{array}$ & $\begin{array}{c}21 . \\
8\end{array}$ & $\begin{array}{c}13 . \\
3\end{array}$ & $\begin{array}{c}140 \\
.5\end{array}$ & 1580 & $\begin{array}{l}4- \\
13\end{array}$ & $\begin{array}{c}23 . \\
7\end{array}$ & $\begin{array}{c}13 . \\
8\end{array}$ & $\begin{array}{c}152 \\
.5\end{array}$ & $\begin{array}{c}1543 \\
.5\end{array}$ \\
\hline & $\begin{array}{l}14- \\
15\end{array}$ & $\begin{array}{c}30 . \\
5\end{array}$ & $\begin{array}{c}17 . \\
5\end{array}$ & 41 & $\begin{array}{c}1812 \\
.5\end{array}$ & $\begin{array}{l}9- \\
15\end{array}$ & $\begin{array}{l}27 . \\
14\end{array}$ & $\begin{array}{c}15 . \\
2\end{array}$ & $\begin{array}{c}123 \\
.7\end{array}$ & $\begin{array}{c}1668 \\
.7\end{array}$ & $\begin{array}{c}14- \\
15\end{array}$ & $\begin{array}{c}30 . \\
5\end{array}$ & $\begin{array}{c}17 \\
5\end{array}$ & $\begin{array}{c}102 \\
.5\end{array}$ & 1646 \\
\hline
\end{tabular}

Table 4. Mean 10day monthly, temperature, growing degree days (GDD) and accumulated growing degree days (AGDD) during barley growing season.

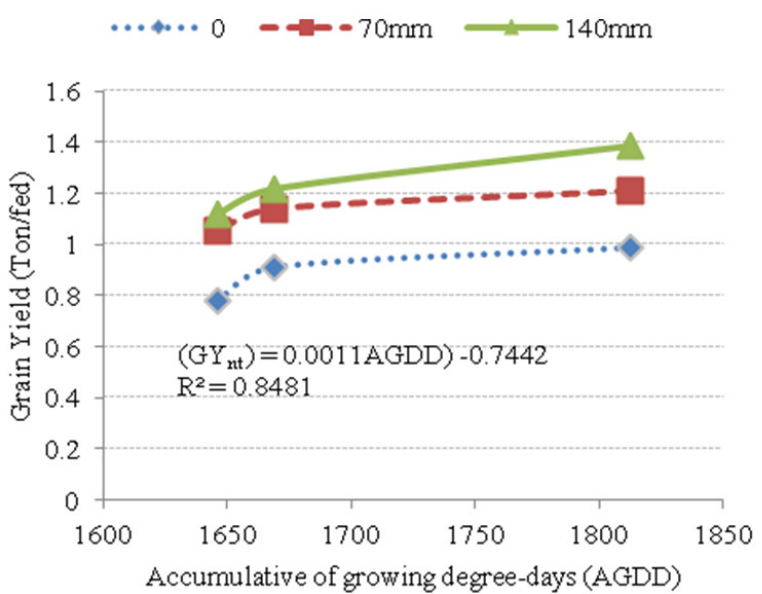

Tillage

Figure 3

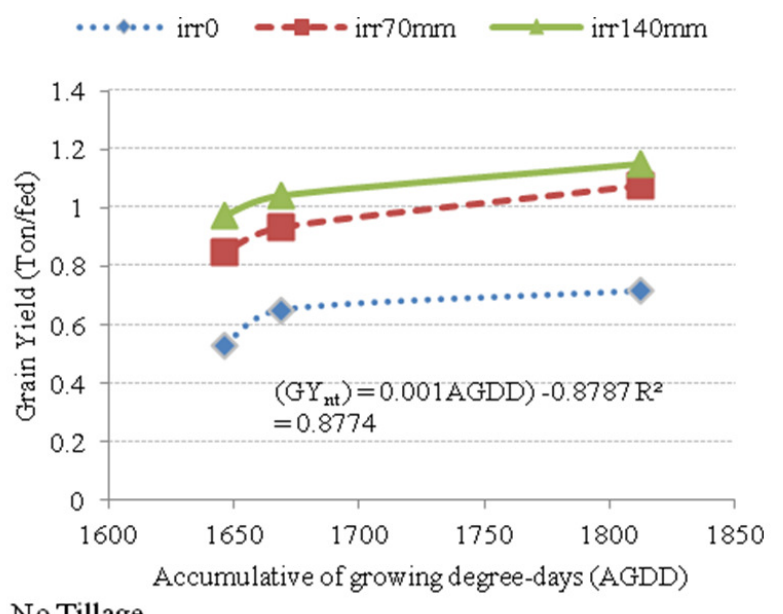

No Tillage

Moreover; data represented at (fig.3). There is a power response between Grain yield and accumulative 
growing degree-days with an average $\mathrm{R}^{2}=0.85$ at both two tillage application treatments (Eq. $9 \& 10$ ).

$$
\mathrm{GY}_{\mathrm{nt}}=0.001(\mathrm{AGDD})-0.8787
$$

Where: -

$\mathrm{GY}_{\mathrm{nt}} \quad=$ The Biomass yield for wheat under No tillage $\left(\right.$ Ton. fed $\left.{ }^{-1}\right)$.

AGDD = Accumulative of growing degree-days $\left(\mathrm{c}^{\circ}\right.$ day $)$

$$
\mathrm{GY}_{\mathrm{nt}}=0.0011(\mathrm{AGDD})-0.7442
$$

Where: -

$\mathrm{GY}_{\mathrm{nt}} \quad=$ The Biomass yield for wheat under tillage $\left(\right.$ Ton. fed $\left.{ }^{-1}\right)$.

AGDD = Accumulative of growing degree-days $\left(\mathrm{c}^{\circ}\right.$ day $)$.

The strong liner relationship between Grain yield and AGDD with average bower $\left(\mathrm{R}^{2}\right)=0.85$ for both treatment tillage and no tillage. Nevertheless; the highest value recorded with tillage treatment where the value of grain yield was $\left(1.4\right.$ Ton.Fed $\left.^{-1}\right)$ under $\left(1812.5 \mathrm{c}^{\circ} /\right.$ season $)$ with $(140 \mathrm{~mm})$ treatment. Generally; with No tillage the $\left(1\right.$ Ton.Fed $\left.^{-1}\right)$ grain yield needs for $(1845.1,1904.2$ and 2099.5$)$ heat units under different sowing dates $\left(15^{\text {th }}\right.$ Nov, $30^{\text {th }}$ Nov and $15^{\text {th }}$ Dec.) respectively with different water treatments. However; under Tillage application (1 Ton.Fed $\left.{ }^{-1}\right)$ from grain yield needs for (1518) heat units if sowing on the (15 ${ }^{\text {th }}$ Nov.) and for (1531.8) heat units if sowing on $\left(30^{\text {th }}\right.$ Nov.) but with sowing date $\left(15^{\text {th }}\right.$ Dec.) the $\left(1\right.$ Ton.Fed $\left.^{-1}\right)$ from grain yield needs for $(1673.3)$ heat units under such conditions.

Furthermore; data of heat uses efficiency (HUE) were obtained as shown on table (5) under different treatments. The highest value observed with $(140 \mathrm{~mm})$ and tillage operation under sowing date $15^{\text {th }}$ Nov by $(2.06 \&$ $0.764 \mathrm{Kg}$. fed ${ }^{-1} \mathrm{C}^{-1} \mathrm{day}^{-1}$ ) for both Biomass and grain yield respectively. Because , the efficiency of utilization of heat in terms of dry matter accumulation, depends on crop type, genetic factors and sowing time and has great practical application ( Rao et al , 1999). In addition; under no tillage operation the same result was recorded with amount of water $(140 \mathrm{~mm})$ and sowing date $\left(15^{\text {th }} \mathrm{Nov}\right)$ by $\left(1.75 \mathrm{Kg}\right.$. fed ${ }^{-1} \mathrm{C}^{-1}$ day $\left.^{-1}\right)$ for biomass and by $(0.63 \mathrm{Kg}$. fed $\left.^{-1} \mathrm{C}^{-1} \mathrm{day}^{-1}\right)$ for grain. However; the lowest value for HUE observed under sowing date $\left(15^{\text {st }}\right.$ Dec. $)$ under both treatments no tillage and tillage operation by $\left(0.973\right.$ and $1.21 \mathrm{Kg}^{-}$fed $\left.^{-1} \mathrm{C}^{-1} \mathrm{day}^{-1}\right)$ for biomass and by $(0.321$ and $0.47 \mathrm{Kg}$. fed ${ }^{-1} \mathrm{C}^{-1}$ day $\left.^{-1}\right)$ respectively under $(0 \mathrm{~mm})$. Ultimately; the best interlaced impact for treatments is using tillage operation with sowing date $15^{\text {th }}$ Nov with adding $(140 \mathrm{~mm})$ as a Supplementary irrigation. Because; that the tillage operation gave an opportunity to collecting more water on surface soil which helping crop at first stage. A purport; good soil water management in rain fed agriculture can also be achieved through minimum tillage and rainwater harvesting techniques /structures (Walter et al, 2006). Further; the heat units at the mid of November may more effective on plantation and phonology for barely comparing with other sowing date.

\begin{tabular}{|c|c|c|c|c|c|c|}
\hline \multirow{4}{*}{$\begin{array}{l}\text { water } \\
\text { treatments } \\
(\mathrm{mm})\end{array}$} & \multicolumn{6}{|c|}{ No Tillage } \\
\hline & \multicolumn{2}{|c|}{$15^{\text {th }}$ Nov. } & \multicolumn{2}{|c|}{$30^{\text {th }}$ Nov. } & \multicolumn{2}{|c|}{$15^{\text {th }}$ Dec. } \\
\hline & Biomass & Grain & Biomass & Grain & Biomass & Grain \\
\hline & \multicolumn{6}{|c|}{$\left(\right.$ Kg. fed ${ }^{-1} \mathrm{C}^{-1}$ day $\left.^{-1}\right)$} \\
\hline 0 & 1.052 & 0.394 & 1.06 & 0.4 & 0.973 & 0.321 \\
\hline 70 & 1.578 & 0.6 & 1.40 & 0.56 & 1.287 & 0.514 \\
\hline 140 & 1.75 & 0.63 & 1.64 & 0.62 & 1.453 & 0.6 \\
\hline \multirow{4}{*}{$\begin{array}{l}\text { water } \\
\text { treatments } \\
\quad(\mathrm{mm})\end{array}$} & \multicolumn{6}{|c|}{ Tillage } \\
\hline & \multicolumn{2}{|c|}{$15^{\text {th }}$ Nov. } & \multicolumn{2}{|c|}{$30^{\text {th }}$ Nov. } & \multicolumn{2}{|c|}{$15^{\text {th }}$ Dec. } \\
\hline & Biomass & Grain & Biomass & Grain & Biomass & Grain \\
\hline & \multicolumn{6}{|c|}{$\left(\right.$ Kg. fed ${ }^{-1} \mathrm{C}^{-1}$ day $\left.^{-1}\right)$} \\
\hline 0 & 1.324 & 0.544 & 1.378 & 0.545 & 1.21 & 0.47 \\
\hline 70 & 1.732 & 0.668 & 1.773 & 0.683 & 1.688 & 0.628 \\
\hline 140 & 2.068 & 0.764 & 2.031 & 0.73 & 1.889 & 0.680 \\
\hline
\end{tabular}

Table 5. Heat use efficiency (HUE) for barely under different treatments

\section{Calibration and validation of Aqua Crop:- Biomass and Grain yield}

As shown at (fig.4). The data indicated that there is a strong relationship between a simulated and measured Biomass Yield (BY) $\left(\mathrm{R}^{2}=0.98 \& \mathrm{E}=0.95 \& \mathrm{RMSE}=11.7 \%\right)$ for barely under different treatments of (sowing dates and supplementary irrigation strategies) with tillage process. Moreover; under No tillage treatment data represented that the relationship between a simulated and measured are still have a good performance for $\left(\mathrm{R}^{2}, \mathrm{E}\right.$ and RMSE) by 0.98 and 0.96 and $9.4 \%$ respectively to Biomass Yield under different treatments. Obviously; from (fig.5) under tillage and no tillage treatments there are an intense relationship between a simulated and measured Grain Yield $(\mathrm{GY})$ by $\left(\mathrm{R}^{2}=0.9 \& \mathrm{E}=0.51 \& \mathrm{RMSE}=11.8 \%\right)$

With tillage process and $\left(\mathrm{R}^{2}=0.89 \& \mathrm{E}=0.75 \& \mathrm{RMSE}=9.8 \%\right)$ with no tillage for barely under different treatments of (sowing dates and supplementary irrigation strategies). Noticeable; that the higher $\mathrm{R}^{2}$ and $\mathrm{E}$ 
values and the lower RMSE values indicated a good model performance. Consequently; these results suggest that the Aqua Crop model is useful for simulating for BY and barely GY under different planting dates, and irrigation strategies.

Finally; after made a statistical analysis for recording data of biomass yield to barley under different factors (Table. 6). Analysis data represented that there are a significant influence for all treatments on biomass yield. The highest value recorded with tillage operation, sowing date $15^{\text {th }}$ Nov and supplementary irrigation (140mm) by 2.85 , 2.85 and 3.09(Ton.Fed ${ }^{-1}$ ) respectively. Further; the same result was observed with Grain yield where there are significant impacts between two treatments tillage and supplementary irrigation. However; under sowing date there is not significant influence. In addition; the best values observed with treatment $\left(140 \mathrm{~mm}\right.$, sowing date $15^{\text {th }}$ Nov. and tillage) by $\left(1.13,1.07\right.$ and 1.07 Ton.Fed $\left.^{-1}\right)$ respectively. These results obtained because the winter wheat needs to some soil managements to enhance soil ability to harvest rain water which reflected on yield production. This agree with (Hatfield et al, 2001) who suggested that it was possible to increase crop by $25-40 \%$ through soil management. On the other hand; that the barley needs to irrigate by limitation Supplementary irrigation to increase both biomass and Grain (K.PSingh and VijayKumar, 1981) pointed out; indicate the possibilities of considerable saving of water $(100 \mathrm{~mm})$ for barley without any decrease in grain yield and increased water-use efficiency (WUE) of wheat and barley by irrigating at critical stages.
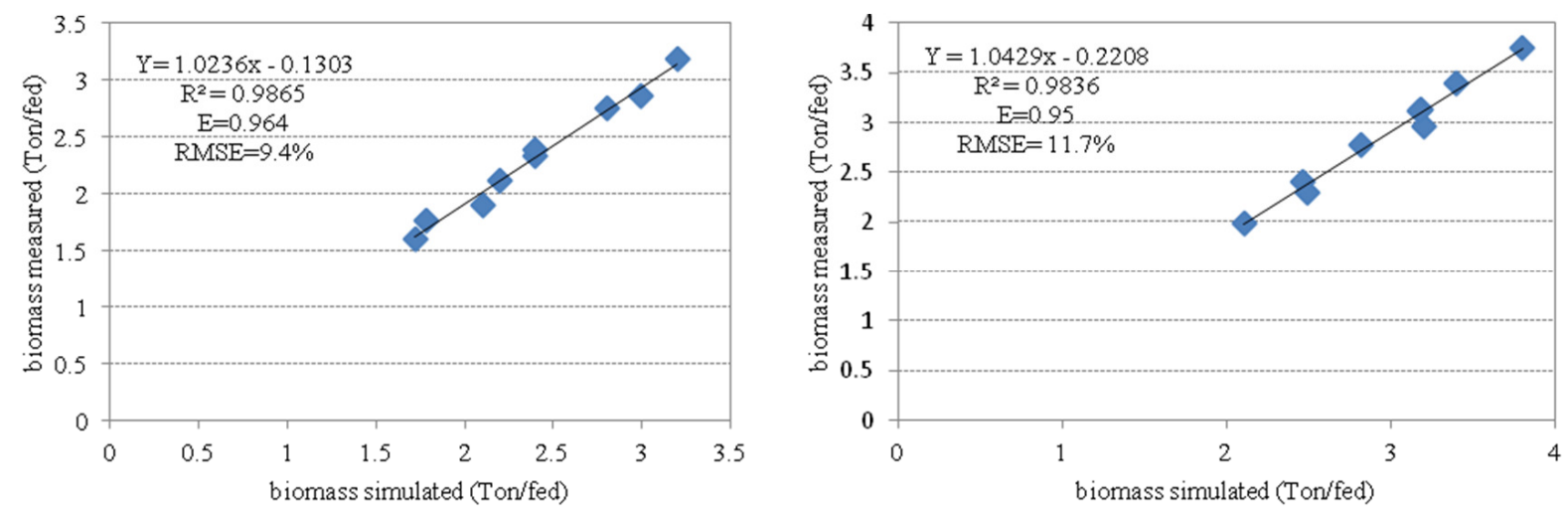

No Tillage

Tillage

Figure 4. Relationship between measured and simulated Biomass Yield (BY) for Barley under different treatments


o Tillage

Tillage

Figure 5. Relationship between measured and simulated Grain Yield (GY) for Barley under different treatments

Table 6. Statistical analysis for all treatments on Biomass and Grain yield for Barley

\begin{tabular}{|c|c|c|c|c|c|c|c|}
\hline \multicolumn{3}{|c|}{ Irrigation } & \multicolumn{3}{|c|}{ Date } & \multicolumn{2}{|c|}{ Tillage operation } \\
\hline $0 \mathrm{~mm}$ & $70 \mathrm{~mm}$ & $140 \mathrm{~mm}$ & $15^{\text {th }} \mathrm{Nov}$ & $30^{\text {th }}$ Nov & $15^{\text {th }}$ Dec. & Tillage & No tillage \\
\hline \multicolumn{8}{|c|}{ Biomass Yield (Ton.Fed $\left.{ }^{-1}\right)$} \\
\hline $1.98 \mathrm{c}$ & $2.67 \mathrm{~b}$ & $3.09 \mathrm{a}$ & $2.85 \mathrm{a}$ & $2.57 \mathrm{~b}$ & $2.31 \mathrm{c}$ & $2.85 \mathrm{a}$ & $2.31 \mathrm{~b}$ \\
\hline \multicolumn{3}{|c|}{ LSD $.05=0.144$} & \multicolumn{3}{|c|}{ LSD .05 $=0.170$} & \multicolumn{2}{|c|}{ LSD $.05=0.086$} \\
\hline \multicolumn{8}{|c|}{ Grain Yield (Ton.Fed $\left.{ }^{-1}\right)$} \\
\hline 0.771 & 1.02 & $1.13 \mathrm{a}$ & $1.07 \mathrm{a}$ & $0.98 \mathrm{a}$ & $0.86 a$ & $1.07 \mathrm{a}$ & $0.873 b$ \\
\hline \multicolumn{3}{|c|}{ LSD $.05=0.081$} & \multicolumn{3}{|c|}{ LSD $.05=0.223$} & \multicolumn{2}{|c|}{ LSD $.05=0.106$} \\
\hline
\end{tabular}




\section{CONCLUSION}

This paper elucidate that the (1 Ton.Fed $\left.{ }^{-1}\right)$ biomass for barely needs $(683.7,729.7$ and 807.6) heat units (AGDD) as an average under different sowing dates $\left(15^{\text {th }}\right.$ Nov, $30^{\text {th }}$ Nov and $15^{\text {th }}$ Dec.) respectively with no tillage treatment. Moreover; the (1 Ton.Fed $\left.{ }^{-1}\right)$ from barely grain yield needs for $(1845.1)$ heat units if sowing on the $\left(15^{\text {th }}\right.$ Nov.) and for (1904.2) heat units if sowing on $\left(30^{\text {th }}\right.$ Nov.) but with sowing date $\left(15^{\text {th }}\right.$ Dec.) the $\left(1\right.$ Ton.Fed $\left.^{-1}\right)$ from grain yield needs for (2099.5) heat units under no tillage treatment. Notable; that the highest value for heat uses efficiency (HUE) observed with $(140 \mathrm{~mm})$ and tillage operation under sowing date $15^{\text {th }}$ Nov by $(2.068 \& 0.784$ $\mathrm{Kg}$. fed ${ }^{-1} \mathrm{C}^{-1} \mathrm{day}^{-1}$ ) for both (BY) and (GY) respectively. Further; the Aqua Crop model adequately simulated the biomass yield (BY), and grain yield (GY) of barely under different sowing dates, irrigation strategies and tillage operation. The simulated (BY) agreed well with the measured (BY) across different treatments. $\left(\mathrm{R}^{2}=0.98 \& \mathrm{E}=\right.$ $0.95 \&$ RMSE $=11.7 \%$ ) for barely under different treatments of (sowing dates and supplementary irrigation strategies) with tillage process. The measured and simulated (GY) were also closely related. The AquaCrop model calibrated the GY with the prediction error statistics of by $\left(\mathrm{R}^{2}=0.9 \& \mathrm{E}=0.51 \& \mathrm{RMSE}=11.89 \%\right)$ with tillage process. Consequently; these results suggest that the AquaCrop model could be used to predict BY and GY of barely with a high degree of reliability at western north coast conditions. Further; that there is a considerable results on barely yield production especially with earlier sowing date $15^{\text {th }}$ Nov under different experiment treatments.

\section{REFERENCE}

Ash, G. H. B., Shaykewich, C. F., and Raddatz, R. L. (1993). The biologically important thermal character of the Eastern Prainie climate. Bulletin Climatologically, 27(1), 3-20.

Baskerville, G. L. and Emin, P. (1969). Rapid estimation of heat accumulation from maximum and minimum temperatures. Ecological Society of America, 50(3), 514-517.

Dirk Raes, Pasquale Steduto, Theodore c. Hsiao, and Elias Fereres.(2011). FAO cropwater productivity model to simulate yield response to water. Reference Manual, Chapter 1 - AquaCrop, Version 3.PP 5-15

Doorenbos, J., \& Kassam, A. H. (1979). Yield response to water. Rome, Italy: FAO Irrigation and Drainage Paper 33, FAO.

FAO AQUASTAT. FAO's Information System on Water and Agriculture: Climate Information tool. AQUASTAT Climate characteristics. http://www.fao.org/nr/water/aquastat/main/ (August 2018)

Geerts S, Raes D, Gracia M, Miranda R, Cusicanqui JA. (2009) Simulating yield response of Quinoa to water availability with AquaCrop. Agronomy Journal. 101: 499-508.

Hatfield, J. L., Thomas, J. S., and John, H. P. (2001). Managing soil to achieve greater water use efficiency: a review. Agronomy Journal, 93, 271-280.

Hsiao TC, Heng L, Steduto P, Roja-Lara B, Raes D. (2009) AquaCrop- The FAO model to simulate yield response to water: parametrization and testing for maize. Agronomy Journal. 101: 448-459.

Johannes S., Jörg S., Undine S., and Ottfried D. (2015). Expansion of an Existing Water Management Model for the Analysis of Opportunities and Impacts of Agricultural Irrigation under Climate Change Conditions.water , 7(11),6351-6377

Kalra, N., Chakraborty, D., Sharma, A., Rai, H. K., Jolly, M., Chander, S., et al. (2008). Effect of increasing temperature on yield of some winter crops in northwest India. Current Science, 94(1), 82-88.

Kingra, P. K., and Prabhjyot-Kaur. (2012). Effect of dates of sowing on thermal utilization and heat use efficiency of groundnut cultivars in central Punjab. Journal of Agricultural Physics, 12(1), 54-62.

Klute, A. (1986). Water retention: laboratory methods. In A. Klute (Ed.), Methods of soil analysis. Part 1. Physical and mineralogical methods (2nd ed.). USA: Am. Soc. Agron., Inc. Soil Sci. Soc. Am (Agronomy; No.9).

K.PSingh and VijayKumar (1981).Water use and water-use efficiency of wheat and barley in relation to seeding dates, levels of irrigation and nitrogen fertilization, Agricultural Water Management Volume 3, Issue 4, July 1981, Pages 305-316

Morteza E. N., Abdolmaahdi B., Mohammad H. G. and Afshin S. (2015). Quantification of Barley Emergence to Temperature. International Journal of Agriculture Innovations and Research, 3 (4), PP.1318-1321

Nash, J. E. and J. V. Sutcliffe (1970), River flow forecasting through conceptual models part I — A discussion of principles, Journal of Hydrology, 10 (3), 282-290.

Penning de Vries FWT, Jansen DM, Berge ten HFM, Bakema A (1989) Simulation of Ecophysiological process of growth in several annual crops. Simulation Monographys, Pudoc, Wagenningen, pp. 82-96.

Raes D, Steduto P, Hsiao TC and Fereres E (2009) AquaCrop-The FAO Crop Model to Simulate Yield Response to Water: Reference Manual Annexes., www. fao.org/nr/water/aquacrop.html.

Rao, V. U. M., Singh, D., and Singh, R. (1999). Heat use efficiency of winter crops in Haryana. Journal of Agrometeorology, 1(2), 143-148.

Snyder, R., Spano, D., Cesaraccio, C., and Duce, P. (1999). Determining degree-day thresholds from field observations. International Journal of Biometeorology, 42, 177-182.

Steduto P, Hsiao TC, Raes D, Fereres E (2009) AquaCrop-The FAO crop model to simulate yield response to 
water. I. Concepts. Agronomy Journal. 101: 426-437.

Walter Mupangwa, David Love and Steve Twomlow (2006).Soil-water conservation and rainwater harvesting strategies in the semi-arid Mzingwane Catchment, Limpopo Basin, Zimbabwe. Physics and Chemistry of the Earth Parts A/B/C; 31(15-16):893-900. DOI: 10.1016/j.pce.2006.08.042

Xiu-liang Jin, Hai-kuan Feng, Xin-kai Zhu, Zhen-hai Li, Sen-nan Song, Xiao-yu Song, Guijun Yang, Xin-gang $\mathrm{Xu}$ and Wen-shan Guo (2014).Assessment of the AquaCrop Model for Use in Simulation of Irrigated Winter Wheat Canopy Cover, Biomass, and Grain Yield in the North China Plain. PLOS ONE. 9; 1:1-11 